\section{Myocysticercosis of the Masseter Muscle}

Sir,

The infestation by the larval stage of the pork tapeworm Taenia solium causes cysticercosis. 1 Cysticerci are spherical, milky white cysts containing fluid and a single invaginated scolex. The most common location of the cyst includes subcutaneous tissues, brain, and skeletal muscles. ${ }^{2}$ However, cysticercal involvement of the masseter muscle is an uncommon and rare presentation and often difficult to diagnose clinically.2,3 In this report, we like to highlight the use of ultrasonography (USG) and computed tomography (CT) scan for diagnosis of extra-oral swelling with painful jaw movements without any obvious dentoalveolar cause.

We report a case of 22-year male patient, who complained of pain in the preauricular region, which increased on mastication and opening of the mouth. Extra-oral examination showed ovoid, freely mobile, solitary, firm tender swelling present on the right side of the face. The swelling was situated $1 \mathrm{~cm}$ above the angle of mandible and $1.5 \mathrm{~cm}$ below the right ear lobe measuring approximately $1 \times 1 \mathrm{~cm}$ in size. The overlying skin appeared normal and freely mobile; but when the patient was asked to clench his teeth, the swelling became fixed, suggesting an involvement of masseter muscle. Intra-oral examination showed no significant findings. Systemic examination of the patient was normal, and no other swelling was noted in any other parts of the body.

The patient was referred for USG examination and CT scan. USG report showed inflammation in right masseter muscle with evidence of two small cystic lesions deep within the muscle with attached visible echogenic and calcified eccentrically placed scolex measuring $3 \times 2 \mathrm{~mm}$. The cystic lesion showed well defined margins and the largest lesion measuring approximately $9 \times 7 \times 8 \mathrm{~mm}$ (Figure 1A). Focal probe elicited tenderness over the swelling. The CT scan report correlated with USG finding, showing thin walled small cystic lesion with a tiny eccentric calcification, mild peripheral enhancement and edema surrounding right masseter muscle (Figure 1B).

The patient was treated conservatively with tablet albendazole (400 mg twice daily for one month) and for symptomatic relief of pain tablet aceclofenac $(100 \mathrm{mg}$ twice daily for one week) was prescribed. The patient was recalled at every 10 days for three months. The

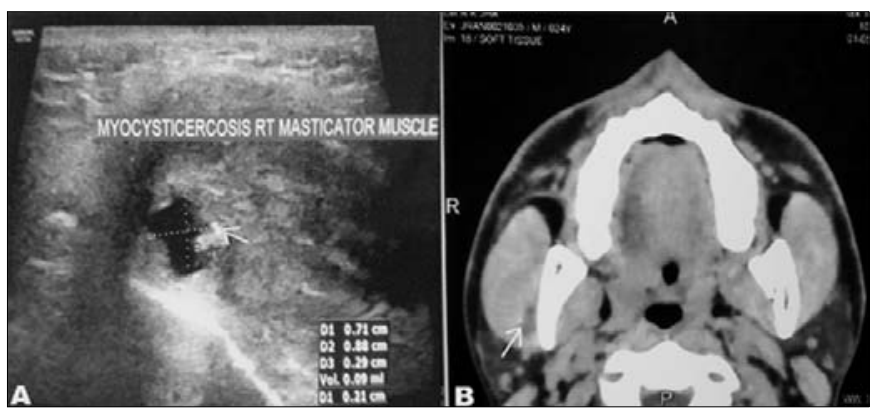

Figure 1: (A) USG showing two small cystic lesions deep within the right masseter muscle. Arrow pointing towards the eccentrically placed calcified scolex. (B) CT scan showing cystic lesion in masseter muscle.

swelling and pain was reduced after 20 days. On followup visit, a repeat USG was performed after 60 days, which showed no abnormality in the right masseter muscle.

This case presented with a diagnostic dilemma to the dental practitioner; and myocysticercosis was diagnosed incidentally after sonographic examination. Therefore, the dental practitioners should be aware of myocysticercosis in cases of extra-oral swelling. Sonography provides valuable information to the likely nature of disease. ${ }^{4}$ Long term discomfort and disability can easily be avoided if the disorder is timely suspected, diagnosed and managed.

\section{REFERENCES}

1. Bhat V, Nagarjuna M, Belaval V, Shetty $S$, Salins PC. Cysticercosis of the masseter: MRI and sonographic correlation. Dentomaxillofac Radiol 2015; 44:20140372.

2. Chaurasia RN, Jaiswal S, Gautam D, Mishra VN. Masseter muscle cysticercosis: A common disease with uncommon presentation. BMJ Case Rep 2013; 2013:bcr2013009127

3. Mittal A, Das D, lyer N, Nagaraj J, Gupta M. Masseter cysticercosis - A rare case diagnosed on ultrasound. Dentomaxillofac Radiol 2008; 37:113-6.

4. Gokarn A, Gokarn S, Rathod V. Ultrasonography for masseter muscle cysticercosis. Indian Pediatr 2011; 48:141-3.

Sonia Nath ${ }^{1}$, Virendra Kumar Prajapati² and Shaju Jacob Pulikkotil ${ }^{3}$

1 Department of Dentistry / Oral and Maxillofacial Surgery2, Dental Institute, Rajendra Institute of Medical Sciences, Ranchi, India

3 Department of Restorative Dentistry, School of Dentistry, International Medical University, Kuala Lumpur, Malaysia

Correspondence: Dr. Sonia Nath, Dental Institute, Rajendra Institute of Medical Sciences, Bariatu, Ranchi 834009, India E-mail: sonianath_12@yahoo.co.in

Received: March 05, 2018; Accepted: October 17, 2018

$$
\text { …ㄷ…. }
$$

